\title{
Thermal spike in muon implantation
}

\author{
R C Vilão, ${ }^{1, \text { f }} \mathrm{H}$ V Alberto, ${ }^{1} \mathrm{~J} \mathrm{M}$ Gil,${ }^{1}$ and A Weidinger ${ }^{2}$ \\ ${ }^{1}$ CFisUC, Department of Physics, University of Coimbra, P-3004-516 Coimbra, Portugal \\ ${ }^{2}$ Helmholtz-Zentrum Berlin für Materialien und Energie, \\ Institute for Nanospectroscopy, D-12489 Berlin, Germany
}

(Dated: May 16, 2019)

\begin{abstract}
In muon spin rotation experiments, the diamagnetic fraction observed at low temperatures is a consequence of the muon interaction with the lattice during the incorporation process and depends on the properties of the material. An increase of the diamagnetic fraction with decreasing temperature is observed in some cases, which we attribute to a thermal spike at the end of the muon trajectory. The thermal spike is due to energy liberated not only during the muon stopping process but also as a consequence of the stress release when the electron bound to a muon in a unrelaxed configuration jumps to a neighboring lattice site. The magnitude and time extension of this effect is more significant for highly disturbed lattices which exhibit very low thermal conductivities at low temperatures. A phenomenological description of some selected examples is presented, together with a discussion about heat transport, using existing models for the thermal spike.
\end{abstract}

PACS numbers: 76.75.+i, 71.55.-i, 61.72.-y, 71.38.-k

\section{INTRODUCTION}

The method of muon spin rotation $(\mu \mathrm{SR})$ spectroscopy ${ }^{11} 4$ uses implanted muons to investigate the local environment around the muon. In materials' science the positive muon, which behaves similarly to a light positive hydrogen ion, is mostly used. The use of negative muons, which behave similarly to a heavy electron, is far more restricted. Two main muon signals can be distinguished, depending on whether a spin interaction between the muon and an unpaired electron is present (paramagnetic signal) or absent (diamagnetic signal) 5 [ The diamagnetic signal stems, strictly speaking, from the bare muon $\mu^{+}$or from the negatively charged bound muonium state $\mathrm{Mu}^{-}$, but the latter is typically restricted to highly doped materials at high temperatures only. ${ }^{7 / 9 / 10}$ In some cases, a muon interacting with an unpaired electron in the vicinity may give rise to a signal with a precession frequency close to the Larmor precession. This is caused by fluctuations of a hyperfine interaction with a typical value in the $\mathrm{MHz}$ range, averaging to a very small value. In those cases, the signal is usually also called diamagnetic, although the designation diamagnetic-like would be more appropriate (see Ref. 11 for a detailed discussion).

The $\mu \mathrm{SR}$ method has proven to be of invaluable value in addressing several problems in condensed matter physics otherwise unacessible by other techniques. Among the most fruitful results of microscopic characterization of solids using muons as probes is the direct measurement of internal fields distributions in magnets and superconductors ${ }^{12}$ or the observation of shallowdonor hydrogen centers in semiconductors ${ }^{13 \mid 14}$ Recently, muons have been proposed as probes of polaron centers in dielectric materials ${ }^{15 \mid 16}$ or of the elusive defect layer in semiconductor interfaces. 17

However, the $\mu \mathrm{SR}$ method relies on the implantation of positive muons at high energies (typically $4 \mathrm{MeV}$ in usual surface beams, although this can be reduced to a few keV in the "slow muons" beam available at the Paul Scherrer Institut) $\frac{18}{18}$ The thermalization process is usually treated as a quick process (on the nanosecond or sub-nanosecond range) with a negligible impact on the muon spin polarization. Despite its importance for a correct understanding of the final observable states, it remains little understood, due to the scarcity of experimental data for its characterization (see Ref. 19 and references therein).

We have however recently showed the presence of a diamagnetic-like muonium state in the final stage of the thermalization (a transition state) that may be observed and impact deeply in the interpretation of $\mu \mathrm{SR}$ data. 11 The formation of this transition state precedes the formation of the final stable state(s) and characteristic barrier(s) result in the final yields. We proposed the transition state model as an alternative to a previously proposed mechanism for the formation of the final states, based on an electric-field effect in $\mu \mathrm{SR}$ experiments showing the presence of a weakly-bound muonium state.20121 In Ref. 11] we discussed both mechanisms and decided for the formation of a (weakly bound) transition state over delayed electron capture from the ionization track.

Moreover, in several recent experiments (e.g. in the dielectric oxide $\mathrm{Lu}_{2} \mathrm{O}_{3}, \frac{19}{19}$ in solar cell materials, $\frac{17}{,}$ or in alloyed $\mathrm{Ge})^{22}$ an increase of the diamagnetic fraction with decreasing temperature, at low temperatures, has been observed. We suggest that this effect is related to the deposited energy - a thermal spike - at the end of the muon trajectory in implantation experiments. Such "thermal spike" not only represents a measurable effect of heat transfer in the microscopic scale, but also opens the door for using $\mu \mathrm{SR}$ as a probe for microscale heat transfer processes. In this paper, the temperature dependence of the diamagnetic fraction will be discussed in more detail for the following three different materials: 


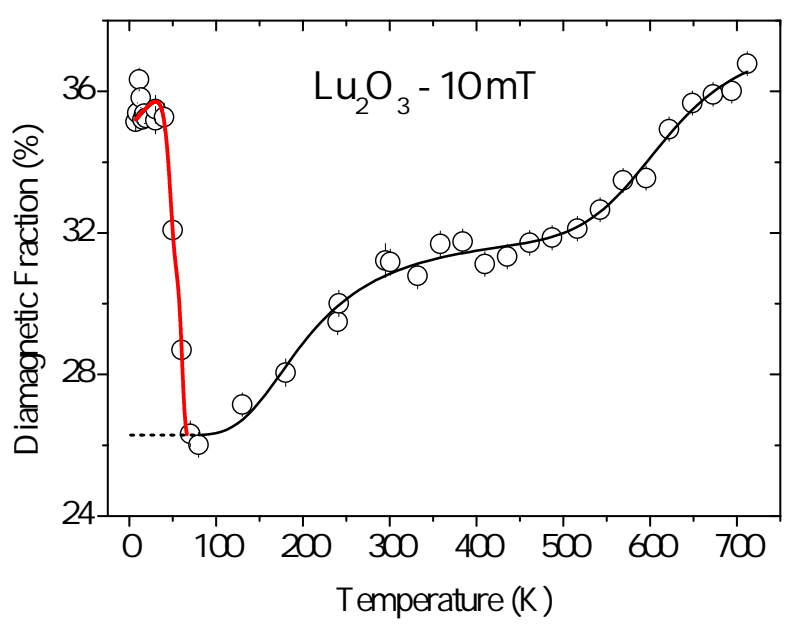

FIG. 1: Temperature dependence of the diamagnetic fraction of $\mathrm{Lu}_{2} \mathrm{O}_{3}$ at a transverse field of $B=10 \mathrm{mT}$ (data from Ref. 19). The black line fit was obtained using a two component Boltzmann recovery function. ${ }^{19}$ The red line is a fit with an effective thermal spike temperature (see text).

\section{1. $\mathrm{Lu}_{2} \mathrm{O}_{3}$}

$\mathrm{Lu}_{2} \mathrm{O}_{3}$ was investigated within the context of the study of the impact of the hydrogen impurity in the electrical properties of dielectric oxides ${ }^{23}$ In this experiment, $\frac{19}{19} \mathrm{a}$ recovery of the diamagnetic fraction with increasing temperature from about $100 \mathrm{~K}$ upward is observed ((Fig. 1)). This is a feature often observed in dielectric materials and is interpreted as a thermally activated process over a barrier (see Ref. 19 and references therein). In this case, the recovery occurs in two-steps, as discussed in detail in Ref. 19. However, at temperatures below about $70 \mathrm{~K}$ the diamagnetic fraction increases with decreasing temperature (red line in Fig. 1). This effect is attributed to the thermal spike, as discussed below.

\section{2. $\mathrm{Cu}_{2} \mathrm{ZnSnS}_{4}(\mathrm{CZTS})$}

The study of $\mathrm{Cu}_{2} \mathrm{ZnSnS}_{4}$ was motivated by its use in thin film solar cells! ${ }^{17}$ At temperatures above about $150 \mathrm{~K}$ the usual recovery of the diamagnetic fraction is observed (Fig. 2). At lower temperatures, a significant difference between the different samples shows up: in the bulk sample the diamagnetic fraction stays constant, whereas in the films a considerable increase is observed. We attribute this effect to the fast disappearance of the thermal spike effect in bulk samples (within a nanosecond timescale), whereas for film samples, where the lattice is somewhat disturbed, the effect lasts for longer times.

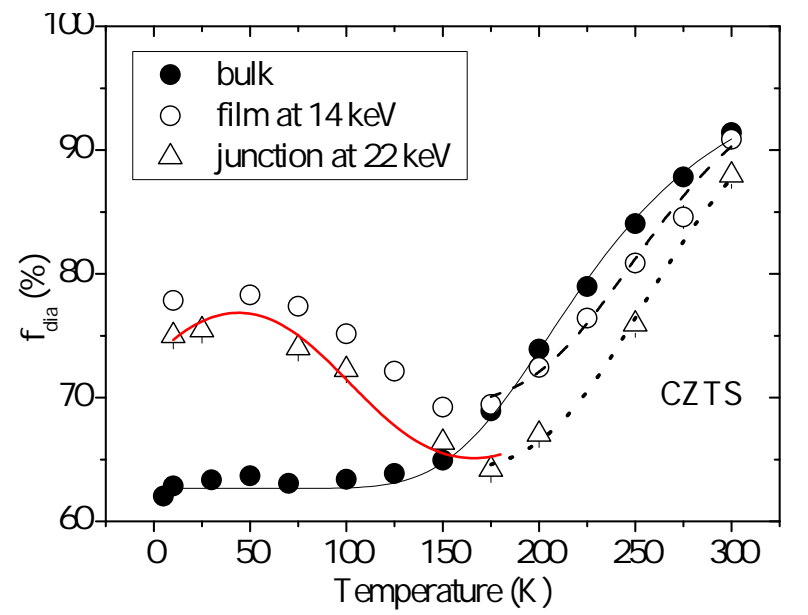

FIG. 2: Temperature dependence of the diamagnetic fraction, $f_{\text {dia }}$, of CZTS (data from Ref. 17). The designation film at $14 \mathrm{keV}$ refers to measurements in which the muons stop in a CZTS film, with an implantation energy of $14 \mathrm{keV}$, whereas junction at $22 \mathrm{keV}$ refers to measurements in a CdS/CZTS junction where a muon implantation energy of $22 \mathrm{keV}$ forces the muon to stop in CZTS, near the interface with CdS. The black curves describe the increase of the diamagnetic fraction above $150 \mathrm{~K}$ as a thermally-activated process. The red line is a fit with an effective spike temperature (see text) for the junction sample.

\section{Ge-rich SiGe alloys}

Figure 3 is reproduced from Ref. 22 and shows longitudinal field data of Ge-rich SiGe alloys. Three different $\mu \mathrm{SR}$ signals are distinguished and named based on their different relaxation (fast, moderate and slow). The interpretation of the signals will be discussed below, but here we would like to note that the low temperature part looks like a distorted mirror image of the high temperature part, as if the low temperature scale had been compressed; the inversion point is around $70 \mathrm{~K}$ (vertical yellow dashed line in Fig. 3p. As in the previous cases, the increase of the fractions with decreasing temperature at low temperatures is attributed to the thermal spike.

We suggest, as a tentative interpretation of the three fractions presented in Fig. 3, that all three signals correspond to muonium in a transition state $\frac{11}{11}$ which is an intermediate configuration between the compact muonium originally formed in the unrelaxed lattice and the final relaxed configuration. The transition state may be considered as a shallow-donor like configuration with a weakly bound electron and an almost vanishing average hyperfine interaction, resulting in diamagnetic-like frequency behavior, as discussed in Ref. 11. The three signals are assigned to i) the ionized configuration (slow) or to conversion of the transition state to ii) atomic muonium (fast) or to iii) bound muonium (moderate), respectively. The final states are not observed in this case due to dephasing, except the ionized one. 


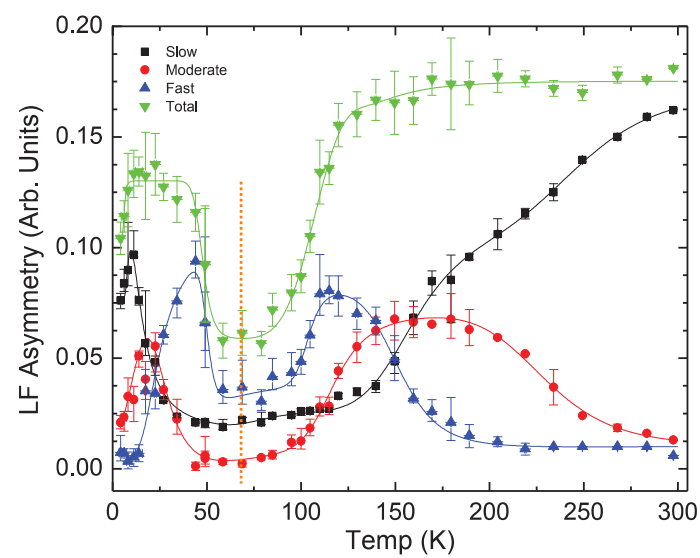

FIG. 3: Temperature dependence of the asymmetry in a longitudinal field experiment on $\mathrm{Si}_{0.09} \mathrm{Ge}_{0.91}$. Slow, moderate and fast refer to different relaxation rates. Total asymmetry, as shown, is calculated by summing the individual components. The low temperature part looks like a mirror image of the high temperature part with a compressed temperature scale. The dashed yellow line was added by us and indicates the approximate inversion point. Reprinted from Ref. 22, with permission from Elsevier.

Mengyan et al. give a different interpretation of the low-temperature signals, 22 they attribute them to an acceptor-like configuration which is formed by the pickup of an electron from the host, forming a $\mathrm{Mu}^{-}$configuration with a diamagnetic frequency behavior. The additional pickup of a hole $\left(\mathrm{h}^{+}\right)$leads to a weakly paramagnetic acceptor configuration with a similar $\mu \mathrm{SR}$ behavior as the proposed donor-like transition state in our model. Experimentally, the two interpretations (donor or acceptor configuration) cannot be distinguished. The thermal spike concept is applicable to both models.

\section{THERMAL SPIKE MODEL}

As discussed in the literature (Refs. 11 and 19 and references therein), positive muons impinging on a target (such as a crystalline dielectric material) are slowed down by various excitation processes, including cyclic charge exchange, and come to rest as bare muon $\mu^{+}$or as compact neutral muonium $\mathrm{Mu}^{0}$ in the basically unrelaxed host lattice. We assume that the positive $\mu^{+}$reacts immediately with the lattice and forms a bound configuration. In contrast, the reaction of neutral muonium $\mathrm{Mu}^{0}$ with the lattice requires overcoming a barrier and needs a source of energy for activation.

The normal reaction of muonium with the lattice is activated by the lattice temperature and leads to an increase of the diamagnetic fraction at higher temperatures (indicated by the solid black lines in Fig. 1 and Fig. 2 and the increase of the total fraction above $100 \mathrm{~K}$ in Fig. 30. There are two additional possible sources of energy for this activation, besides the lattice temperature. One is the potential energy of muonium in the unrelaxed lattice: as the atom-like muonium in the final stage of the thermalization process stops in an interstitial site in the lattice, some stress is released as the electron jumps to a nearby position some distance away from the muon. This mechanism was suggested in the interpretation of zirconia data, $\frac{16}{16}$ where the experimental observations, together with theoretical calculations, ${ }^{24}$ are consistent with an electron transfer from muonium to a $\mathrm{Zr}$ atom, reducing it from $\mathrm{Zr}^{4+}$ to $\mathrm{Zr}^{3+}$.

A second contribution to the thermal spike stems from the excitation of a lattice vibration caused by the final stopping of the muon. These two sources of energy (the potential energy of the constrained muonium and the contribution of the kinetic energy) are responsible for a thermal spike at the end of the muon trajectory.

\section{A. The phenomenological model}

The thermal spike at the end of the muon trajectory triggers a reaction between atom-like muonium and the lattice. This could be a prompt transition to the final states, but it may also involve a transition to an intermediate state where the electron is weakly bound to the muon. We call this state transition state or weakly bound state 11

The efficiency of the formation of the transition state depends not only on the maximum effective temperature at the muon positions, but also on the duration of the thermal spike. The thermal spike disappears due the spread of the local spike energy into the surroundings and the speed of this dissipation depends on the thermal conductivity of the material. This quantity is not always well known at low temperatures but it is usually lower for less perfect materials (such as alloys or materials with defects or with a microcrystalline structure), becoming very small at low temperatures. ${ }^{25}$ Thus, the thermal spike effect is expected only at low temperatures and mainly for imperfect materials.

The spike temperature is characterized by a saturation value at low temperature (when the heat spike stays near the muon site) and a gradual decrease with increasing temperature and can therefore be described by an inverted S-function (also called sigmoid or logistic function):

$$
T_{\text {spike }}(T)=\frac{T_{0}}{1+\exp \left[k\left(T-T_{1 / 2}\right)\right]}
$$

where $T_{0}$ represents the maximum spike temperature and $T_{1 / 2}$ is such that $T_{\text {spike }}\left(T_{1 / 2}\right)=T_{0} / 2 ; k$ is a parameter that determines the rate of temperature decrease. The effective temperature felt by the muon is given by: $T_{\text {eff }}=T_{\text {spike }}+T$.

The total diamagnetic fraction, $f_{d i a}$, corresponding to the sum of all diamagnetic-like fractions (fast, moderate 
and slow) is calculated using the Boltzmann distribution:

$$
f_{\text {dia }}=f_{\text {min }}+\left(f_{\text {max }}-f_{\text {min }}\right) \frac{N \exp \left(-\frac{E_{b}}{k_{B} T_{e f f}}\right)}{1+N \exp \left(-\frac{E_{b}}{k_{B} T_{e f f}}\right)}
$$

Here $N$ is a statistical weight factor, $E_{b}$ is the barrier height, $k_{B}$ is the Boltzmann constant and $T_{\text {eff }}$ the effective temperature. The factor $\left(f_{\max }-f_{\min }\right)$ corresponds to the total thermal recovery fraction. $f_{\min }$ represents the contribution from $\mu^{+}$and possibly prompt reaction to the transition state. The parameters $N, E_{b}$, and $\left(f_{\max }-f_{\min }\right)$ can be obtained from the fit to the thermal recovery of the reacted fraction at high temperatures. An equivalent formula holds for the unnormalized asymmetry $a$ used in Fig. 3 and in Fig. 4

It should be mentioned that the spike temperature has not the same meaning as an usual lattice temperature since it does not correspond to a situation of thermodynamical equilibrium with the surroundings: The hot spot can emit phonons to the surroundings but it can not receive phonons at the same rate. Thus, the local temperature changes with time and the major part of the reactions occurs at early times when the local temperature is high. A consequence is that the reaction from compact muonium to the transition state occurs mainly in one direction whereas in thermodynamical equilibrium forth and back reactions are possible. ${ }^{26}$ The rather strong dephasing effect in irreversible reactions may explain the weak diamagnetic fraction in transverse field measurements on $\mathrm{Si} / \mathrm{Ge}$ alloys at low temperatures. 2728

Figure 4 shows an example for the calculation of the diamagnetic fraction with Eq. 1 and Eq. 2. The parameters are chosen to simulate approximately the total amplitude of the $\mathrm{Si}_{0.09} \mathrm{Ge}_{0.91}$ experiment of Ref. 22 , presented in Fig. 3. The two temperature contributions to the effective temperature are shown in the top frame of Fig. 4. The lower frame of Fig. 4 displays the total asymmetry extracted from Fig. 3, as well as the calculated diamagnetic asymmetry with the parameters shown in the insert.

It follows from the previous considerations that the presence of a thermal spike may lead to a pattern of the temperature dependence of the diamagnetic fraction, as is observed in some systems: it starts with a high value at low temperatures, suffers a decrease at intermediate temperatures and comes back at higher temperatures when the thermal conversion sets in.

We have simulated the low-temperature increase of the diamagnetic fraction using Eq. 2. The parameters $N$, $E_{b}$ and the total recovery fraction were kept equal to those obtained for the analysis of the high-temperature increase but the normal temperature was replaced by $T_{\text {eff }}=T_{\text {spike }}+T$ (Eq. 1). The parameters $T_{0}, T_{1 / 2}$ and $k$ in Eq. 1 1 were used as parameters for the simulation and the best values obtained for the three different materials are shown in table IThe obtained corresponding curves for the total diamagnetic-like fraction are shown as red

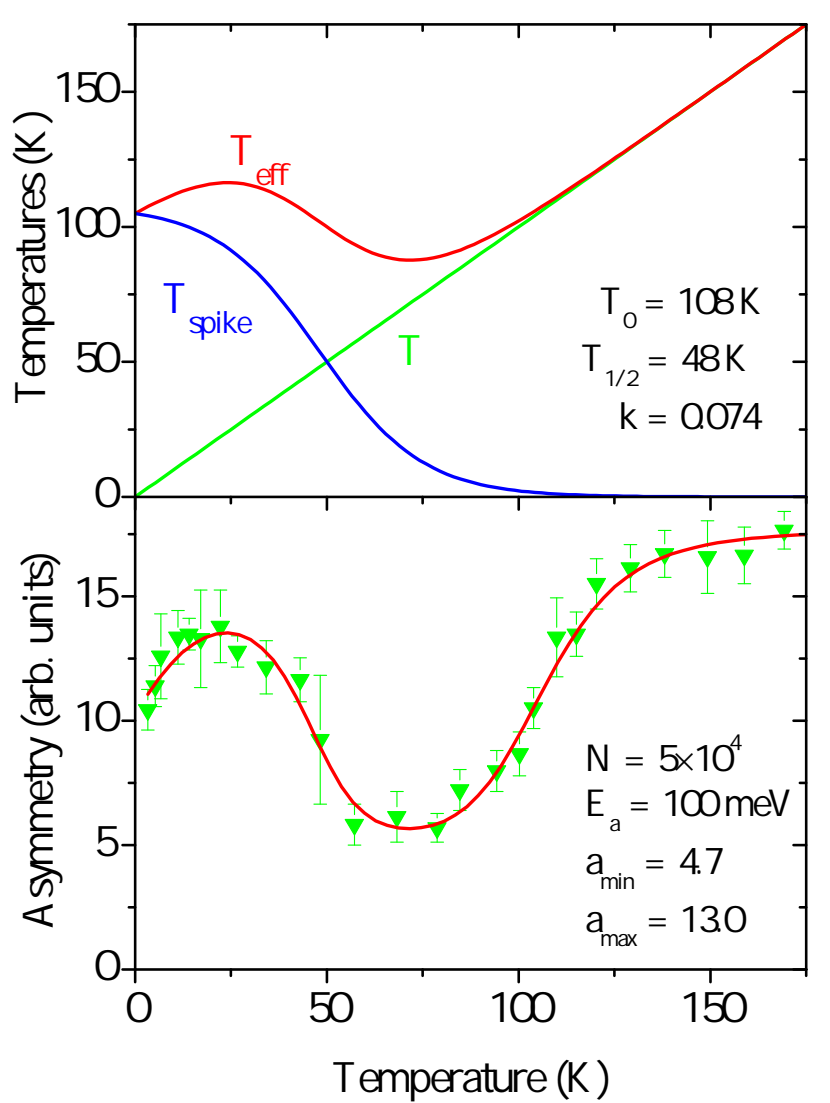

FIG. 4: Simulation of the total asymmetry in the $\mathrm{Si}_{0.09} \mathrm{Ge}_{0.91}$ experiment of Ref. 22 (Fig. 3). Top: The effective temperature at the muon site is a sum of the lattice temperature, $T$, and a local, temperature-like effect $T_{\text {spike }}: T_{\text {eff }}=T_{\text {spike }}+T$. The spike temperature is given by Eq. 1 with the parameters shown in the insert. Bottom: Total asymmetry obtained from Fig. 3 The red line is a simulation of the asymmetry using a total fraction as described in Eq. 2 with the effective temperature $T_{\text {eff }}$ shown in the upper frame. The corresponding parameters describing the thermal recovery above $100 \mathrm{~K}$ are shown in the insert.

lines in Fig. 1 and Fig. 2 A simulation of the total asymmetry is also presented at the bottom frame of Fig. 4 for the complete temperature range.

The value of $\mathrm{T}_{0}$ is expected to depend on the total available energy at the end of the muon trajectory as well as on the transfer of this energy to local vibrations of the surrounding atoms within the order of magnitude observed in table [I A crude estimate of the effect of this thermal spike can be obtained assuming that the total energy of these two processes amounts to around $\Delta E=0.3 \mathrm{eV}$, that this energy is distributed between 10 atoms, and that it leads to an increase in local temperature governed by $\Delta E=3 k_{B} \Delta T / 2$; than an increase $\Delta T \sim 350 \mathrm{~K}$ of the local temperature is estimated. This is of course not a real calculation of the temperature; it is 
TABLE I: Parameters of the thermal spike temperature obtained by fitting Eq. 1 and Eq. 2 to the data of the three selected materials discussed in this paper (Figs. 1 to 3). Fitting errors are of the order of a few percent, but systematic uncertainties are probably larger.

\begin{tabular}{lccc}
\hline \hline Sample & $\mathrm{T}_{0}(\mathrm{~K})$ & $\mathrm{T}_{1 / 2}(\mathrm{~K})$ & $\mathrm{k}$ \\
\hline $\mathrm{Lu}_{2} \mathrm{O}_{3}$ & 653 & 54 & 0.21 \\
$\mathrm{Cu}_{2} \mathrm{ZnSnS}_{4}$ & 277 & 105 & 0.022 \\
(junction structure) & & & \\
$\mathrm{Si}_{0.09} \mathrm{Ge}_{0.91}$ & 108 & 48 & 0.074 \\
\hline \hline
\end{tabular}

just to show what could be roughly expected. $T_{0}$ values in the order of those shown in table [ are expected for most dielectric materials.

In contrast, the values for $T_{1 / 2}$ at which the thermal spike disappears are determined by the thermal conductivity of the material and are expected to become very small for fairly perfect materials with high thermal conductivity, leading to a complete disappearance of the spike effect.

The factor $k$ relates to the slope of the increase of the thermal conductivity with increasing temperature. The very different values of $k$ for the three samples suggest that the onset of heat diffusion is rather abrupt in $\mathrm{Lu}_{2} \mathrm{O}_{3}$, moderate in $\mathrm{Si}_{0.09} \mathrm{Ge}_{0.91}$ and gentle in $\mathrm{Cu}_{2} \mathrm{ZnSnS}_{4}$ (junction structure), although this indication should be taken with caution, given the phenomenological nature of this model.

\section{B. The microscopic model}

After stopping, muonium forms a metastable configuration. A possible reaction, besides the prompt formation of the final states, is the release of the electron to a neighboring position, the electron being still slightly bound by the screened Coulomb potential. Fig. 5 shows a sketch of the suggested potential profile associated with such a rearrangement. The corresponding energy release is expected to depend on the material and is suggested to be the order of a few $100 \mathrm{meV}$, considerably smaller than the full relaxation energy. The total self-trapping energy, liberated when the electron and the muon are in their final positions in the completely relaxed lattice, is likely to be in the order of $1 \mathrm{eV}$ or higher. ${ }^{29}$

In the following we will discuss the thermal spike phenomenon in a model developed for track formation in heavy ion implantation. But before that we would like to mention that the phonon bottleneck effect observed in electron spin relaxation (EPR) experiments ${ }^{30131}$ has similarities with the phenomena observed here; in both cases, a non-equilibrium local heating plays a role. The bottleneck in EPR is due to the fact that the spin-flip transition couples mainly to phonons in a small energy band around the spin-flip energy (bottle-neck) and only

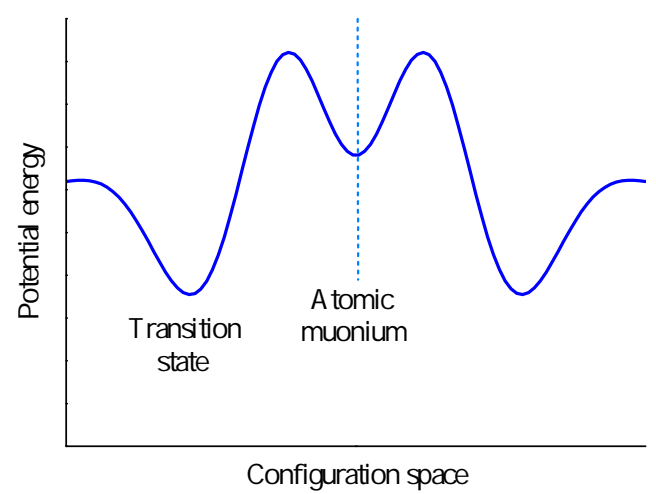

FIG. 5: Suggested schematic potential profile for muonium in the unrelaxed lattice. The energy associated with the compact atom-like configuration around the $\mu^{+}$is higher than the energy of a configuration - which we named as transition state - where the electron is some distance away from the muon (transition state). Note that the transition state energy minima exist in all three spatial directions and also at larger distances, but the nearest positions are favored by the Coulomb attraction, and correspond to the formation of a weakly bound state.

the further decay to other degrees of freedom of the bath leads to thermal equilibration. In the present case the bottle-neck is due to the phonons which are strongly coupled to the constrained muonium electron (this could be e.g. the breathing mode). However, not enough knowledge is available in the present case to make use of models developed in the EPR literature.

The thermal spike model is discussed extensively in the literature in connection with latent ion tracks which are produced by the passage of heavy ions trough matter $\underline{32}$ The ion deposits energy along the ion trajectory due to the slowing-down process. This energy is converted into heat and diffuses into the surrounding. The heat transport is treated with the classical diffusion equation. ${ }^{33}$ We adopt this description here but instead of the stringlike thermal spike in the heavy ion case, we consider the diffusion equation for a point-like source. This threedimensional case is also treated in the Vineyard paper ${ }^{33}$

A general analytical solution of the diffusion equation is not possible. Vineyard presents solutions for the case of power-law temperature dependences of the parameters. In our case the temperature dependence of those parameters is not known. Since the heat transport away from the hot zone is guided by the phonons of the basically unheated lattice, we assume the case of constant conductivity and diffusivity and fix them to the values relevant at the measuring-temperature.

The heat transport away from the thermal spike (assumed to be a delta function in space and time) in the case of an isotropic medium and constant parameters, can be described by: 


$$
c_{\nu} \frac{\partial T}{\partial t}=\kappa \nabla^{2} T, \text { for } \kappa=\text { const. }
$$

Here $\nabla^{2}$ is the Laplace operator and $c_{\nu}$ and $\kappa$ the volume specific heat and the thermal conductivity, respectively.

The solution of the diffusion equation (Eq. 3 ) yields the typical bell-shape distribution of the temperature $T$ as a function of time and radial distance, $T(r, t)$, for constant parameter values $: \underline{33}$

$$
T(r, t)=\frac{Q}{(4 \pi)^{3 / 2} \kappa \sqrt{D}} t^{-3 / 2} \exp \left(-\frac{r^{2}}{4 \pi D t}\right)
$$

where $Q$ is the heat input, $D=\kappa / c_{\nu}$ the thermal diffusivity and $\kappa$ the thermal conductivity. In the present context, the pre-exponential factor of Eq. 44, i.e. the temperature at the muon site $T(r=0, t)$, is of interest. From this expression, we expect that the smaller the diffusivity and the conductivity, the longer the duration of the thermal spike at the muon site, and thus the probability for a reaction.

It is important to keep in mind that the assumption of a point-like source at $t=0$ is not realistic. It is more likely that the energy initially available is distributed among vibrations of the surrounding atoms which then initiate the reaction. Nevertheless, for a qualitative discussion, the first factor in Eq. 4 may be considered as a measure of the reaction probability. Therefore, the temperature and material dependence of the reaction probability can be discussed using the parameterization of $D$ and $\kappa$ presented in the literature,$\frac{25}{25}$ namely:

$$
D=\frac{1}{3} v_{g} l \text { and } \kappa=\frac{1}{3} c_{\nu} v_{g} l
$$

where $v_{g}$ is the sound velocity, $l$ the mean free path of phonons and $c_{\nu}$ the volume specific heat. Thus, the first factor in Eq. 4 becomes

$$
\frac{Q}{(4 \pi)^{3 / 2} \kappa \sqrt{D}}=0.12 \frac{Q}{v_{g}^{3 / 2} l^{3 / 2} c_{\nu}}
$$

It is important to note that the parameters $c_{\nu}$ and $l$ are strongly temperature and sample dependent but that the same does not apply for $v_{g}{ }^{34}\left(v_{g}\right.$ values for the present

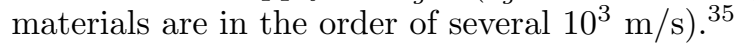

\section{Sample dependence}

The sample dependence is dominated by the behaviour of the phonon mean free path parameter, $l$. At low temperatures, $l$ is determined mainly by defects and becomes very small for highly disturbed lattices and thus favors a longer stay of the spike energy at the muon site.
This is evident for the Ge sample where the thermal spike is observed for the alloyed material (see Fig. 3) but not for well order normal Ge $\stackrel{36 \mid 37}{ }$ Low dimensional structures like thin films or nanostructured powders also limit the mean free path ${ }^{25}$ In the present CZTS example (Fig. 2), the thermal spike effect is seen for thin film CZTS but not in the corresponding bulk material (see Fig. 2). The $\mathrm{Lu}_{2} \mathrm{O}_{3}$ sample which shows the thermal spike effect (Fig. 1) was a polycrystalline powder. Thus, the thermal spike effect (see Eq. 6) is expected only for disturbed or dimension-limited samples. The three selected examples discussed in this paper belong to this category.

\section{Temperature dependence}

The temperature dependence is mainly governed by the specific heat. At low temperatures, the specific heat becomes very small and approaches zero at $T=0 \mathrm{~K}$, which, according to Eq. 4 and Eq. 6, should cause the initial temperature at $r=0$ to become infinity at $T=0$. This is obviously not the case, since the heat is not confined to a single point but is distributed in a small area. Thus, even without heat diffusion, the initial temperature remains finite and reaches a plateau value at low temperatures, as discussed previously in the phenomenological model. As the temperature increases, the specific heat also increases and has a strong impact on the thermal conductivity of the sample. The thermal spike disappears when the thermal conductivity becomes sufficiently large to transport the heat away from the muon site in a short time (that is, in a nanosecond time scale).

\section{CONCLUSIONS}

We have discussed some example of $\mu \mathrm{SR}$ experiments where a re-increase of the diamagnetic-like fraction has been observed with decreasing temperature, at low temperatures. This phenomenon can be explained as due to a thermal spike at the end of the muon trajectory. The spike energy is partially due to the stopping process itself but also to the stress energy released from atomic muonium in the unrelaxed lattice, namely when the muonium electron leaves the confined atomic configuration and jumps to a neighboring position. This energy release mimics a local temperature increase around the muon. The duration of this temperature spike, and thus of the reaction probability, depends on the properties of the host material and on its temperature. A discussion about heat transport using existing models for the thermal spike, suggests that the thermal spike duration is significant only in disturbed materials and at low temperatures, where the thermal conductivity and 
diffusivity are small. ${ }^{25}$ Thus the thermal spike effect is expected to be observed only in imperfect lattices and at low temperatures, such as in the case of the examples discussed in this paper. This effect may be important not only for the discussion and interpretation of $\mu \mathrm{SR}$ data in general, but it may also represent a novel means to address microscopically the important problem of microand nano-heat transfer in disordered solids.

\section{ACKNOWLEDGEMENTS}

This work was supported with funds from the Portuguese National Budget through Fundação para a Ciência e Tecnologia/MCTES and also from the European Regional Development Fund (ERDF) through the Competitiveness and Internationalisation Operational Programa (PT-COMPETE 2020), under projects UID/FIS/04564/2016 and PTDC/FIS$\mathrm{MAC} / 29696 / 2017$.
* ruivilao@uc.pt

1 P. J. C. King, R. de Renzi, S. P. Cottrell, A. D. Hillier, and S. F. J. Cox, ISIS muons for materials and molecular science studies, Physica Scripta 88, 068502 (2013).

2 S. F. J. Cox, R. L. Lichti, J. S. Lord, E. A. Davis, R. C. Vilão, J. M. Gil, T. D. Veal, and Y. G. Celebi, The first 25 years of semiconductor muonics at ISIS, modelling the electrical activity of hydrogen in inorganic semiconductors and high- $\kappa$ dielectrics, Physica Scripta 88, 068503 (2013).

3 S. F. J. Cox, Muonium as a model for interstitial hydrogen in the semiconducting and semimetallic elements, Reports on Progress in Physics 72, 116501 (2009).

4 Page of the Laboratory for muon spectroscopy of the Paul Scherrer Institut in the internet, https://www.psi.ch/lmu/ (2019).

5 A. Weidinger, J. M. Gil, H. V. Alberto, R. C. Vilão, J. Piroto Duarte, N. Ayres de Campos, and S. F. J. Cox, Shallow donor versus deep acceptor state in II-VI semiconductor compounds, Physica B: Condensed Matter 326, 124 (2003).

${ }^{6}$ S. F. J. Cox, J. L. Gavartin, J. S. Lord, S. P. Cottrell, J. M. Gil, H. V. Alberto, J. Piroto Duarte, R. C. Vilão, N. Ayres de Campos, D. J. Keeble, E. A. Davis, M. Charlton, and D. P. van der Werf, Oxide muonics: II. Modelling the electrical activity of hydrogen in wide-gap and highpermittivity dielectrics, Journal of Physics: Condensed Matter 18, 1079 (2006).

7 R. C. Vilão, J. M. Gil, A. Weidinger, H. V. Alberto, J. Piroto Duarte, N. Ayres de Campos, R. L. Lichti, K. H. Chow, S. P. Cottrell, and S. F. J. Cox, Acceptor level of interstitial muonium in ZnSe and ZnS, Physical Review B 77, 235212 (2008).

8 R. C. Vilão, A. G. Marinopoulos, R. B. L. Vieira, A. Weidinger, H. V. Alberto, J. P. Duarte, J. M. Gil, J. S. Lord, and S. F. J. Cox, Hydrogen impurity in paratellurite $\alpha-\mathrm{TeO}_{2}$ : Muon-spin rotation and ab initio studies, Phys. Rev. B 84, 045201 (2011).

${ }^{9}$ K. H. Chow, B. Hitti, R. F. Kiefl, S. R. Dunsiger, R. L. Lichti,and T. L. Estle, Diffusion and Charge Dynamics of Negatively Charged Muonium in n-Type GaAs, Physical Review Letters 76, 3790 (1996).

${ }^{10}$ R. C. Vilão, J. M. Gil, A. Weidinger, H. V. Alberto, J. Piroto Duarte, and B. F. O. Costa, N. Ayres de Campos, R. L. Lichti, K. H. Chow, S. P. Cottrell, and S. F. J.
Cox, Delayed electron capture and $\mathrm{Mu}^{-}$formation in $\mathrm{ZnSe}$, Physica B: Condensed Matter 404, 888 (2009).

11 R. C. Vilão, R. B. L. Vieira, H. V. Alberto, J. M. Gil, and A. Weidinger, Role of the transition state in muon implantation, Phys. Rev. B 96, 195205 (2017).

12 P. Carretta, R. de Renzi, G. Prando, and S. Sanna, A view from inside iron-based superconductors, Physica Scripta 88, 068504 (2013).

13 J. M. Gil, H. V. Alberto, R. C. Vilão, J.P. Duarte, P. J. Mendes, L. P. Ferreira, N. Ayres de Campos, A. Weidinger, J. Krauser, Ch. Niedermayer, and S. F. J. Cox, Novel Muonium State in CdS, Phys. Rev. Lett. 83, 5294 (1999).

14 S. F. J. Cox, E. A. Davis, S. P. Cottrell, P. J. C. King, J. S. Lord, J. M. Gil, H. V. Alberto, R. C. Vilão, J. Piroto Duarte, N. Ayres de Campos, A. Weidinger, R. L. Lichti, and S. J. C. Irvine, Experimental Confirmation of the Predicted Shallow Donor Hydrogen State in Zinc Oxide, Phys. Rev. Lett. 86, 2601 (2001).

15 R. C. Vilão, R. B. L. Vieira, H. V. Alberto, J. M. Gil, A. Weidinger, R. L. Lichti, B. B. Baker, P. W. Mengyan, and J. S. Lord, Muonium donor in rutile $\mathrm{TiO}_{2}$ and comparison with hydrogen, Physical Review B 92, 081202(R) (2015).

16 R. B. L. Vieira, R. C. Vilão, A. G. Marinopoulos, P. M. Gordo, J. A. Paixão, H. V. Alberto, J. M. Gil, A. Weidinger, R. L. Lichti, B. Baker, P. W. Mengyan, and J. S. Lord, Isolated hydrogen configurations in zirconia as seen by muon spin spectroscopy and $a b$ initio calculations, Phys. Rev. B 94, 115207 (2016).

17 H. V. Alberto, R. C. Vilão, R. B. L. Vieira, J. M. Gil, A. Weidinger, M. G. Sousa, J. P. Teixeira, A. F. da Cunha, P. M. P. Salomé, P. A. Fernandes, T. Törndahl, T. Prokscha, A. Suter, and Z. Salman, Slow-muon study of quaternary solar-cell materials: Single layers and $p-n$ junctions, Phys. Rev. Materials 2, 025402 (2018).

18 E. Morenzoni, T. Prokscha, A. Suter, H. Luetkens, and R. Khasanov, Nano-scale thin film investigations with slow polarized muons, Journal of Physics: Condensed Matter 16, S4583 (2004).

19 R. C. Vilão, R. B. L. Vieira, H. V. Alberto, J. M. Gil, A. Weidinger, R. L. Lichti, P. W. Mengyan, B. B. Baker, and J. S. Lord, Barrier model in muon implantation and application to $\mathrm{Lu}_{2} \mathrm{O}_{3}$, Phys. Rev. B 98, 115201 (2018).

${ }^{20}$ V. Storchak, J. H. Brewer, and G. D. Morris, Quantum 
transport of electronic polarons in sapphire, Phys. Rev. B 56, 55 (1997).

${ }^{21}$ V. G. Storchak, D. G. Eshchenko, and J. H. Brewer, Formation and dynamics of muonium centres in semiconductorsa new approach, Journal of Physics: Condensed Matter 16, S4761 (2004).

22 P. W. Mengyan, Y. G. Celebi, R. L. Lichti, B. R. Carroll, B. B. Baker, H. N. Bani-Salameh, and I. Yonenaga, Longitudinal Muon Spin Depolarization in Ge-Rich SiGe Alloys, Physics Procedia 30, 214 (2012).

23 E. L. da Silva, A. G. Marinopoulos, R. B. L. Vieira, R. C. Vilão, H. V. Alberto, J. M. Gil, R. L. Lichti, P. W. Mengyan, and B. B. Baker, Electronic structure of interstitial hydrogen in lutetium oxide from $\mathrm{DFT}+U$ calculations and comparison study with $\mu \mathrm{SR}$ spectroscopy, Phys. Rev. B 94, 014104 (2016).

24 A. G. Marinopoulos, Incorporation and migration of hydrogen in yttria-stabilized cubic zirconia: Insights from semilocal and hybrid-functional calculations, Physical Review B 86, 155144 (2012).

${ }^{25}$ R. Berman, The thermal conductivity of dielectric solids at low temperatures, Advances in Physics 2, 103 (1953).

${ }^{26}$ R. L. Jayarathna, R. L. Lichti, P. W. Mengyan, Y. G. Celebi, B. B. Baker, B. R. Carroll, and I. Yonenaga, Transition dynamics for $\mathrm{Mu}$ acceptor states in $\mathrm{Si}_{1 x} \mathrm{Ge}_{x}$ alloys, AIP Conference Proceedings 1583, 56 (2014).

27 B. R. Carroll, R. L. Lichti, P. J. C. King, Y. G. Celebi, I. Yonenaga, and K. H. Chow, Muonium acceptor states in high Ge $\mathrm{Si}_{1-x} \mathrm{Ge}_{x}$ alloys, Physica B 404, 812 (2009).

28 B. R. Carroll, R. L. Lichti, P. J. C. King, Y. G. Celebi, I. Yonenaga, and K. H. Chow, Muonium defect levels in Czochralski-grown silicon-germanium alloys, Physical Review B 82, 205205 (2010).

29 L. Gordon, H. Abu-Farsakh, A. Janotti, and C. G. Van de Walle, Hydrogen bonds in $\mathrm{Al}_{2} \mathrm{O}_{3}$ as dissipative two-level systems in superconducting qubits, Scientific Reports 4,
7590 (2014).

${ }^{30}$ P. L. Scott, and C. D. Jeffries, Spin-Lattice Relaxation in Some Rare-Earth Salts at Helium Temperatures; Observation of the Phonon Bottleneck, Phys. Rev. 127, 32 (1962).

${ }^{31}$ K. J. Standley, and R. A. Vaughan, The Phonon Bottleneck, Electron Spin Relaxation Phenomena in Solids, Monographs on Electron Spin Resonance, Springer, Boston, MA, (1969).

32 M. Toulemonde, E. Paumier, and C. Dufour, Thermal spike model in the electronic stopping power regime, Radiation Effects and Defects in Solids 126, 201 (1993).

33 G. H. Vineyard, Thermal spikes and activated processes, Radiation Effects: Incorporating Plasma Science and Plasma Technology 29, 245 (1976).

${ }^{34}$ H. Ledbetter, Sound velocities, elastic constants: Temperature dependence, Materials Science and Engineering: A 442, 31 (2006).

35 M. A. Breazeale and M. McPherson, Physical Acoustics, Springer Handbook of Acoustics, edited by T. D. Rossing, Springer, New York (2007),

36 B. Patterson, Reviews of Modern Physics 60, 69 (1988).

37 R. L. Lichti, S. F. J. Cox, K. H. Chow, E. A. Davis, T. L. Estle, B. Hitti, E. Mytilineou, and C. Schwab, Charge-state transitions of muonium in germanium, Phys. Rev. B 60, 1734 (1999).

38 A. Sood, R. Cheaito, T. Bai, H. Kwon, Y. Wang, C. Li, L. Yates, T. Bougher, S. Graham, M. Asheghi, M. Goorsky, and K. E. Goodson, Direct Visualization of Thermal Conductivity Suppression Due to Enhanced Phonon Scattering Near Individual Grain Boundaries, Nano Letters 18, 3466 (2018).

39 F. Kargar, E. H. Penilla, E. Aytan, J. S. Lewis, J. E. Garay, and A. A. Balandin, Acoustic phonon spectrum engineering in bulk crystals via incorporation of dopant atoms, Applied Physics Letters 112, 191902 (2018). 